CARPATHIAN J. MATH.

Volume 38 (2022), No. 2,

Pages 477 - 487

\title{
Analysis of a nonlinear financial model
}

\author{
G. MoZA, E. GRECU and L. TiRTiRAU
}

\begin{abstract}
A three-dimensional nonlinear differential system for modeling inflation rate in a given economy is proposed and investigated in this article. Using a bifurcation-based method in the study, we describe the conditions leading to regions of stability for the long-term behavior of the model, which are economically desirable, along with regions of instability, which should be avoided in an economy.
\end{abstract}

\section{INTRODUCTION}

In this paper we have carried out a reassessment of a studied economic model, taking into account a nonlinear evolution. Thus, compared to the model studied in [10], for calculating the time variation of the inflation rate, we added the effect generated by the economic agents' expectations on the evolution of the real interest rates. In this way, the model becomes

$$
\dot{x}=z+x(y-a), \dot{y}=1-b y-x^{2}, \dot{z}=-x-c z+m x y,
$$

where $x=x(t)$ is the real interest rate (calculated as the difference between the nominal interest rate and the inflation rate), $y=y(t)$ the investment demand, $z=z(t)$ the inflation rate, $a \in \mathbb{R}$ the amount (of money) saved, $b>0$ the cost per investment, $c>0$ the elasticity of the demand on the commercial market [10] and $m>0$ the effect of the anticipations on the evolution of the real interest rate.

The adding of the new term $m x y$ is based on the following facts. The decisions of the economic agents are influenced not only by the objective characteristics of the economicsocial environment in which they operate, but also by their anticipations (expectations) regarding the future, which they make, taking into account all the significant information they have [3]. Anticipations regarding the future evolution of monetary policy interest rates influence a wide spectrum of real rates $x(t)$ of long-term interest rates of economic agents (as well banks and markets). Also, expectations influence investment $y(t)$ and private consumption. For example, if higher profits are anticipated, companies will tend to increase their investment spending. If households expect higher real incomes as a result of increased anticipated labor productivity, they will be inclined to increase their consumer spending [7]. Therefore, in general, strengthening investor and consumer confidence is associated with increased aggregate demand and vice versa in the case of decreased confidence, in the sense of decreased aggregate demand.

The paper is organized as it follows. In the first section we define the model and present links with other financial models. Section 2 is devoted to the mathematical analysis of the model and economic interpretations of the results. It contains five sub-sections. The first one is dedicated to the mathematical analysis of the model around its first equilibrium point, which is followed by a sub-section dealing with economic interpretations of the

Received: 12.03.2021. In revised form: 21.07.2021. Accepted: 28.07.2021

2020 Mathematics Subject Classification. 37N40, 34C23.

Key words and phrases. economic models, dynamical systems, bifurcations.

Corresponding author: G. Moza; gheorghe.moza@upt.ro 
results. Sub-section three is dedicated to the study of the model around the other equilibrium points, followed by sub-section four where we present economic relevance of the results. A discrete-time analysis is presented in sub-section five. The paper ends with conclusive remarks.

\section{ANALYSIS OF THE MODEL}

Denote further by $\alpha=\frac{c+m-a b c-b}{c+m}$. Notice that $c+m \neq 0$. For $\alpha \leq 0$ the system has a single equilibrium point $A\left(0, \frac{1}{b}, 0\right)$, while two more equilibria arise when $\alpha>0$, namely

$$
B\left(\sqrt{\alpha}, \frac{a c+1}{c+m},-\sqrt{\alpha} \frac{1-a m}{c+m}\right) \text { and } C\left(-\sqrt{\alpha}, \frac{a c+1}{c+m}, \sqrt{\alpha} \frac{1-a m}{c+m}\right) .
$$

Remark 2.1. Notice that $B$ and $C$ lie in the same plane $y=y_{0}$, where $y_{0}=\frac{a c+1}{c+m}$, and are symmetrical with respect to the $y$-axis.

\subsection{Properties of the equilibrium $A$.}

Theorem 2.1. The following assertions are true. 1) If $\alpha<0, A$ is an attractor for $1-a b-b c<0$, respectively, a saddle for $1-a b-b c>0$.

2) If $\alpha<0$ and $1-a b-b c=0$, the system (1.1) undergoes a Hopf bifurcation at $A$.

3) If $\alpha>0$, then $A$ is a saddle-node.

Proof. 1) The characteristic equation at $A$ reads

$$
(\lambda+b)\left(b \lambda^{2}-(1-a b-b c) \lambda-\alpha(c+m)\right)=0 .
$$

Thus, the eigenvalues at $A$ satisfy $\lambda_{1}=-b<0$, respectively, $\lambda_{2} \lambda_{3}=-\frac{c+m}{b} \alpha$ and $\lambda_{2}+\lambda_{3}=$ $\frac{1-a b-b c}{b}$. If $\alpha<0, A$ is an attractor on $1-a b-b c<0$ because $\lambda_{1}<0$ and $\operatorname{Re}\left(\lambda_{2,3}\right)<0$, respectively, a saddle on $1-a b-b c>0$ with $\operatorname{dim}\left(W^{s}\right)=1$ and $\operatorname{dim}\left(W^{u}\right)=2$ because $\lambda_{1}<0$ and $\operatorname{Re}\left(\lambda_{2,3}\right)>0$. More exactly, $A$ is a saddle-node if $\Delta>0$, respectively, saddlefocus if $\Delta<0$, where $\Delta=(1-a b-b c)^{2}+4 b \alpha(c+m)$.

2) Assume $a, c, m$ fixed with $a+c>0$ and $m<\frac{1-c^{2}}{c+a}$, and let $b$ varies with $\alpha<0$. Notice that $0<m<\frac{1-c^{2}}{c+a}$ is well-defined for all $c$ with $0<c<1$ and $a+c>0$. Then, at $b=b_{0}$, where $b_{0}:=\frac{1}{a+c}$, the complex eigenvalues at $A$ become $\pm i \omega_{0}$, where $\omega_{0}=\sqrt{1-c^{2}-m(a+c)}>0$. Since $\left.\frac{\partial \operatorname{Re}\left(\lambda_{2,3}\right)}{\partial b}\right|_{b=b_{0}}=-\frac{1}{2}(a+c)^{2} \neq 0$, a Hopf bifurcation occurs at $A$. The bifurcation is non-degenerate if the first Lyapunov coefficient $l_{1}(0) \neq 0$ and degenerate if $l_{1}(0)=0$.

3) It is clear that $A$ is a saddle when $\alpha>0$ since $\lambda_{2} \lambda_{3}<0$. It is also a node because $\lambda_{2} \lambda_{3}<0$ yields $\lambda_{2,3} \in \mathbb{R}$. Thus, $A$ is a saddle-node. Since $\lambda_{1}=-b<0$ and $\lambda_{2}<0$ or $\lambda_{3}<0$, it follows that $\operatorname{dim}\left(W^{s}\right)=2$ and $\operatorname{dim}\left(W^{u}\right)=1$.

Remark 2.2. Since the model (1.1) depends on four parameters $a, b, c, m$, the expression of $l_{1}(0)$ is typically complex. It can be obtained easier in particular cases of the parameters.

Corollary 2.1. Due to the Hopf bifurcation at $1-a b-b c=0$ for $\alpha<0$, when $l_{1}(0) \neq 0$ a unique periodic orbit (limit cycle) arises around the equilibrium $A$. More exactly, when $l_{1}(0)<0$, the limit cycle is stable and exists when $A$ is unstable (saddle-focus), that is for $b<\frac{1}{a+c}$, respectively, when $l_{1}(0)>0$, the limit cycle is unstable and exists when $A$ is attractor, $b>\frac{1}{a+c}$.

Remark 2.3. The existence of a stable periodic orbit around an unstable (saddle-focus) equilibrium point $A$, changes significantly the economic interpretation of the model, as we will see in the following. 
2.2. Economic interpretation of the model around $A$. Four main cases arise depending on the stability of $A$ and the existence of the Hopf bifurcation.

1) Assume $\alpha<0$ and $1-a b-b c<0$, thus, $A$ is an attractor. Then, for any starting value $u_{0}=(x(0), y(0), z(0))$ sufficiently close to $A\left(0, \frac{1}{b}, 0\right)$, the dynamics of the economic system is one that converges towards $A$, that is, $(x(t), y(t), z(t))$ tends to the point $\left(0, \frac{1}{b}, 0\right)$ for $t$ increasing and sufficiently large, which is constituted as a stable (node or focus) stationary state. If a very low real interest rate $x(0)$ and inflation rate $z(0)$ are taken, both around 0 , and the investment demand $y(0)$ is approximately equal to the inverse of the cost per investment $(y(0) \approx 1 / b)$, then the economy will converge to the equilibrium point $A$.

Thus, because the real interest rate $x(t)$ (which tends, in the case of this balance $A$, to $0)$ is given by the difference between the nominal interest rate and the inflation rate $z(t)$ (which tends also to 0 ), it turns out that the nominal interest rate will be equal to 0 . In such a situation, (theoretically) nominal interest rates can no longer be reduced and prices tend to remain constant [6]. A monetary policy that maintains price stability, i.e. $z(t) \rightarrow 0$, generally has positive effects on economic activity. If prices are stable, economic agents (companies and consumers) do not risk misinterpreting the changes in the general level of prices, considering them variations in relative prices, and can make decisions related to consumption and investments in the informed case. From a theoretical point of view, in order to stimulate demand and avoid falling prices, budget deficits should be increased, which is not desirable [11].

On the other hand, when approaching the equilibrium point $A$ in the model, it may appear cases when $x(t)<0$, or even the starting point could be negative, $x(0)<0$. Thus, the model reaches the situation where a negative interest rate policy should be instituted. This is an unusual monetary policy instrument in which nominal interest rates are set at a negative value, below the theoretical lower limit of zero percent. Such a situation is not advisable. "Zero-bound" is an expanding monetary policy tool, where a central bank reduces short-term interest rates to zero, if necessary, to stimulate the economy [9]. "Zerobound" is the lower limit at which rates can be reduced, but not beyond that. When this level is reached and the economy is still under performing, the central bank can no longer provide incentives through interest rates. "Liquidity trap" is the term used by economists to describe this scenario [9]. A liquidity trap is a situation where interest rates are low and savings rates high (which makes monetary policy inefficient) [11]. In a liquidity trap, if a country's central bank tries to stimulate the economy by increasing its money supply, it would have no effect on interest rates (as economic agents should not be encouraged to hold additional cash) [1]. When faced with a liquidity trap, alternative monetary stimulation procedures are often needed for some countries [11]. Conventionally, the idea was that interest rates could not move in the negative territory, which means that, once interest rates reach zero or close to zero, for example, $0.01 \%$, monetary policy must be changed to further stabilizes or stimulating the economy.

Thus, an economic model of type (1.1) which oscillates around a stationary attracting state $A\left(0, \frac{1}{b}, 0\right)$ might be good, this presenting also the scenario with negative interest rate policy.

Figure 1 presents numerically the attracting character of $A(0,1 / b, 0)$ for $a=2.26, c=$ $m=0.5$ and two values of $b$, namely $b=0.5$ and $b=2$. The eigenvalues of $A$ in the first case are $-0.26,-0.5,-0.5$, while in the second $-1.13 \pm 0.59 i$ and -2 . Thus, $A$ is a stable node, respectively, focus in the two cases. We notice that $b>\frac{m+c}{a c+1}$ in order to have $\alpha<0$. Orbits starting close to $A$ are attracted by $A$. 

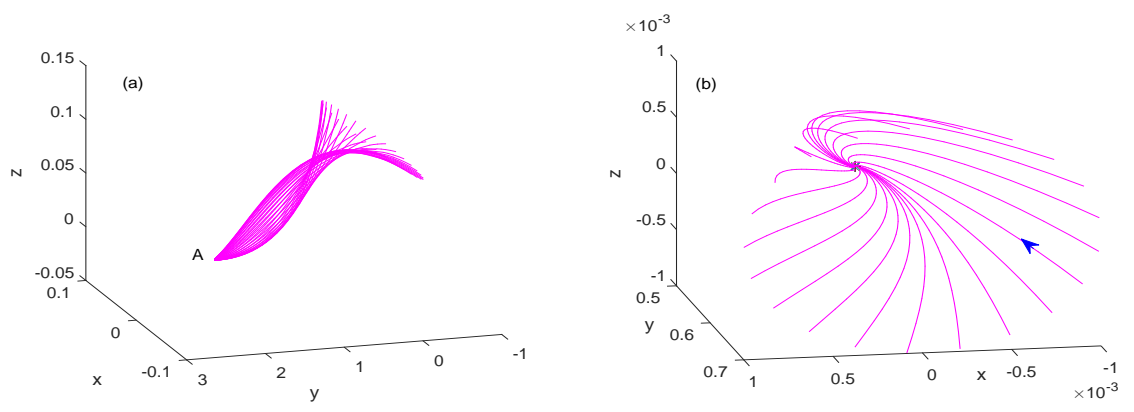

FIGURE 1. The steady state $A$ attracts nearby orbits for $a=2.26, c=0.5, m=0.5$ and (a) $b=0.5$, respectively, (b) $b=2$.

2) Assume further $\alpha<0$ and $1-a b-b c>0$, that is, $\frac{m+c}{a c+1}<b<\frac{1}{a+c}$. We assume also $0<m<\frac{1-c^{2}}{c+a}$ and $l_{1}(0)<0$, in order to have Hopf bifurcation with negative first Lyapunov coefficient. Thus, $A$ is a saddle-focus (unstable) point and, because $l_{1}(0)<0$, a stable periodic orbit (limit cycle) $\Gamma_{1}$ is born around $A$ for all $1-a b-b c>0$ with $\left|b-b_{0}\right|$ sufficiently small; $b_{0}=\frac{1}{a+c}$. Since $\lambda_{1}<0$ and $\operatorname{Re}\left(\lambda_{2,3}\right)>0$, there exist a stable manifold $W^{s}$ of dimension 1 and an unstable manifold $W^{u}$ of dimension 2 , both passing through $A$ and being invariant to the model's flow, that is, an orbit starting on $W^{s}$ (or $W^{u}$ ) remains on $W^{s}$ (or $\left.W^{u}\right)$.

The behaviors of the model on the two manifolds are completely different. If an orbit starts from an initial point $u_{0}=\left(x_{0}, y_{0}, z_{0}\right)$ lying on $W^{s}$, then the orbit $(x(t), y(t), z(t))$ converges to $A\left(0, \frac{1}{b}, 0\right)$ for $t$ increasing, theoretically $t \rightarrow \infty$, while if $u_{0} \in W^{u}$ then the orbit $(x(t), y(t), z(t))$ departs from $A$ but it still stays around $A$ being attracted by the stable limit cycle $\Gamma_{1}$.

Thus, when starting with values from $W^{s}$, the economy will stabilize towards $A$, while starting from $W^{u}$, the economy will have converging tendencies towards the periodic orbit $\Gamma_{1}$. Moreover, starting with $u_{0}$ which is neither on $W^{s}$ nor $W^{u}$ but close to $A$, the corresponding orbit $(x(t), y(t), z(t))$ will converge to $\Gamma_{1}$ for $t$ increasing. Therefore, an unstable saddle-type balance will appear which is surrounded by an island of stability, thus, yielding economic relevance.

Figures 2-3 present numerically this scenario for $a=1, b=0.6, c=0.4$ and $m=0.4$, where $A(0,1.6,0)$ is a saddle-focus with its eigenvalues $0.13 \pm 0.2 i$ and -0.6 , surrounded by a stable periodic orbit. Figure 4 shows the time series of the periodic orbit depicted in Figure 3. We observe from Figure 4 that the periodic orbit enters the zone with negative values for $x(t)$ and $z(t)$.

From an economic point of view, when the model presents this scenario of existing a stable periodic orbit around the unstable steady state $A$, it ofers predictability and, thus, can be recommended [4]. The existence of the stable limit cycle ensures a long-term stability of the economic model because any orbit starting at a point sufficiently close to $\Gamma_{1}$ will oscillate around the limit cycle closer and closer to it or will tend to $A$ [2]. This form of predictability when the behavior of the model approximates a periodic orbit may describe better a real economy (which oscillates in a region of stability rather then tending to a precise steady state) and would be more desirable in practice.

3) Assume $\alpha<0,1-a b-b c>0$ and $l_{1}(0)>0$. This corresponds to the situation when $A$ is a saddle point but no stable periodic orbits exist sufficiently close to $A$. Thus, an orbit starting close to $A$ from a point $u_{0} \notin W^{s}$ will depart from $A$ and may escape to infinity. 

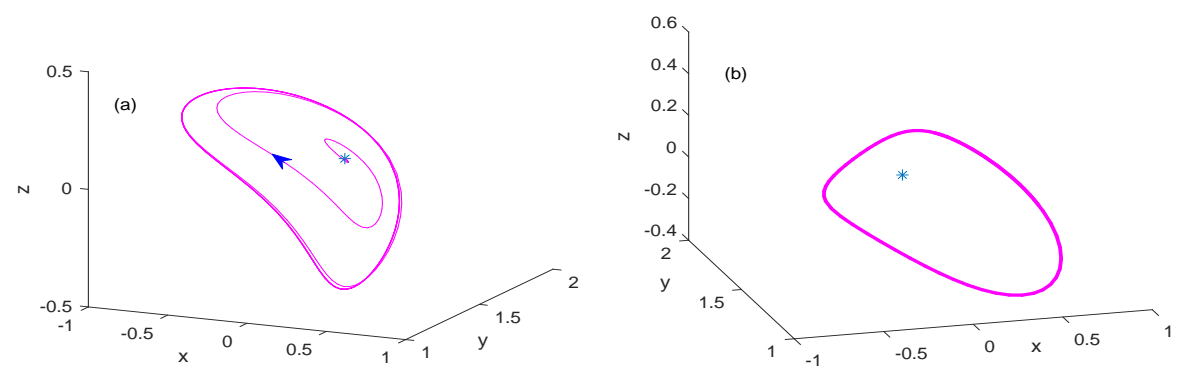

FIGURE 2. The parametric values used in this figure are $a=1, b=0.6, c=0.4, m=0.4$. The figure presents the behavior of an orbit starting close to $A$ (a), respectively, a stable periodic orbit $\Gamma_{1}$ arising around an unstable (saddle-focus) equilibrium $A$ (b); the periodic orbit was obtained with the starting point $(0.033,1.431,0.314)$.
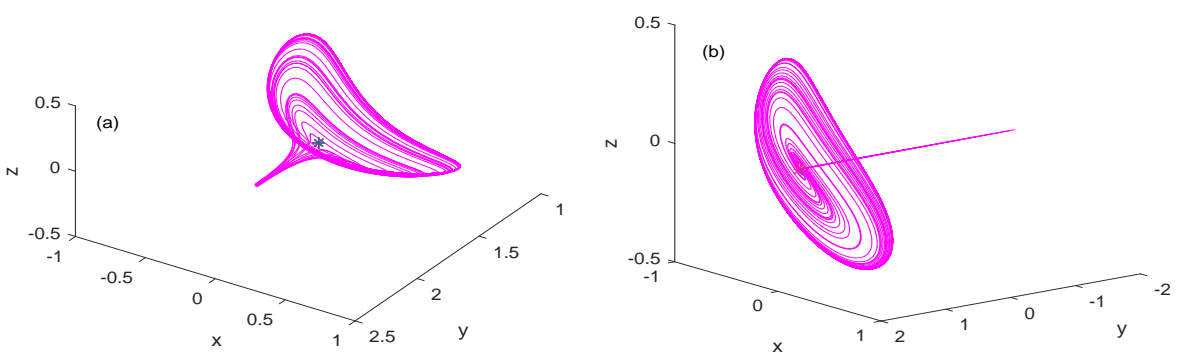

FIGURE 3. A bunch of orbits approaching the stable periodic orbit $\Gamma_{1}$ for $a=1, b=$ $0.6, c=0.4, m=0.4$.

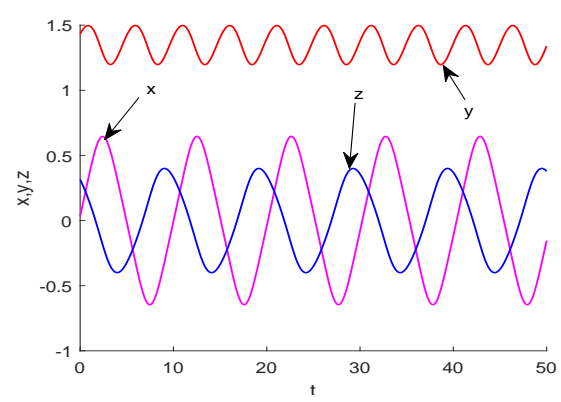

FIGURE 4 . The time series of the periodic orbit starting at $(0.033,1.431,0.314)$ and parametric values $a=1, b=0.6, c=0.4, m=0.4$.

From an economic point of view, this steady state is not advisable in general as we explain below.

On one hand, an increase in real interest rates, as a result of monetary policy measures, will lead to a reduction in current spending in the economy (provided that the other variables would be maintained constant values). This will reduce the attractiveness of current consumption among households and the current investments of companies will be discouraged. On the other hand, lowering real interest rates could lead to a "liquidity trap" whose clear indication is given by low interest rates (and possibly increased savings rates) 
[9]. In order to stimulate demand, real interest rates may fall below 0 and bring them into the negative territory.

Examples of zero and even negative interest rate policies sometimes arise because of the economic turmoil that requires lowering interest rates to stimulate the economy. Negative interest rates can occur during deflationary periods, when businesses have too much money instead of spending. This can lead to a sharp drop in demand and a sharp drop in prices. Under such conditions, reducing the central bank's interest rate to zero is not always sufficient to stimulate credit and lending growth. In recent years, some central banks (from Europe and Japan) have implemented a negative interest rate policy (regarding excess bank reserves in the financial system) [8]. There are cases where negative rates have been implemented in normal periods (economically speaking). For example, in Switzerland in mid-2019, the target interest rate was $-0.75 \%$ to maintain very low (and negative) rates to prevent the currency from growing too significantly (Switzerland is regarded as a country with low inflation and with a currency considered as a safe heaven) [12]. Japan has adopted a similar policy, with a target rate in mid-2019 of $-0.1 \%$. This tool was designed to stimulate economic growth through expenditure and investment [8]. In this way, it has been attempted that depositors should be encouraged to spend cash rather than deposit it at the bank and bear a guaranteed loss (generated by negative real interest).

4) If $\alpha>0$, then $A$ is a saddle-node with $\operatorname{dim}\left(W^{s}\right)=2$ and $\operatorname{dim}\left(W^{u}\right)=1$. The model does not undergo a Hopf bifurcation, thus, no stable periodic orbit is expected around $A$. Following an argument as in 3), the behavior of the model around $A$ does not present economic relevance. However, as we will prove in the next section, when $\alpha>0$ the model presents economic relevance for its behavior around the equilibrium $B$.

2.3. Properties of the equilibrium points $B$ and $C$. We proceed in the following to study the other equilibrium points $B$ and $C$. The characteristic equation at $B$ and $C$ reads

$$
\lambda^{3}+a_{2} \lambda^{2}+a_{1} \lambda+a_{0}=0,
$$

where $a_{2}=a+b+c-\frac{a c+1}{c+m}, a_{1}=2 \alpha+b c+a b-\frac{(a c+1) b}{c+m}$ and $a_{0}=2 \alpha(c+m)$. Denote further by $k_{0}=\left(a m-2 a c+c m+c^{2}-3\right) a_{2}+\left(a_{1}+2 a c+2\right)(c+m)$.

Remark 2.4. Since $\lambda_{1} \lambda_{2} \lambda_{3}=-a_{0}<0$ whenever $B$ and $C$ exist as non-trivial equilibria, i.e. on $\alpha>0, B$ and $C$ cannot be repellers (i.e. $\lambda_{1,2,3}>0$ ).

A long-term financial strategy aims to determine conditions in which an economy remains in one or more zones of stability [2]. For our model, this means to determine sufficient conditions for $B$ and $C$ to be attractors or find stable periodic orbits around $B$ and $C$. In this regard, the following result is obtained.

Theorem 2.2. Assume $\alpha>0$. Then, the following assertions are valid.

1) The equilibrium points $B$ and $C$ are attractors if and only if $a_{2}>0$ and $a_{2} a_{1}>a_{0}$.

2) If $a_{2} a_{1}=a_{0}, a_{1}>0$ and $k_{0} \neq 0$, the system (1.1) undergoes a Hopf bifurcation at $B$ and $C$.

Proof. 1) From Routh-Hurwitz criterion, the characteristic equation (2.2) has all roots with negative real parts if and only if $a_{0}>0, a_{2}>0$ and $a_{2} a_{1}>a_{0}$. Since $a_{0}>0$ whenever $\alpha>0$, the proof is completed. Outside these conditions, $B$ and $C$ may be saddles or nonhyperbolic.

2) The characteristic equation (2.2) has two purely complex roots of the form $\pm i \omega_{0}$ if and only if $a_{1}>0$ and $a_{2} a_{1}=a_{0}$, where $\omega_{0}=\sqrt{a_{1}}$. The equation (2.2) reads in this case $\left(\lambda+a_{2}\right)\left(\lambda^{2}+a_{1}\right)=0$. Notice that this occurs when $a_{2}=a_{0} / a_{1}>0$, thus, the real root at the bifurcation value is negative, $\lambda_{1}=-a_{2}<0$. 
Fix further $a \in \mathbb{R}, c>0$ and $m>0$ while $b$ can vary, thus, $b$ is the bifurcation parameter. We can find $b$ from $a_{2} a_{1}-a_{0}=0$ in the form

$$
b_{0}=\frac{1}{\delta}\left(h_{1} \pm \sqrt{h_{2}}\right),
$$

where $h_{1}=h_{1}(a, c, m)$ and $h_{2}=h_{2}(a, c, m)$ are two smooth functions of their arguments and $\delta=2\left(2 a c-a m-c m-c^{2}+3\right)$, provided that $h_{2} \geq 0$. Thus, $b_{0}$ is the Hopf bifurcation value.

Differentiating with respect to $b$ in (2.2), we get

$$
\left(3 \lambda^{2}+2 a_{2} \lambda+a_{1}\right) \frac{\partial \lambda}{\partial b}+\frac{\partial a_{2}}{\partial b} \lambda^{2}+\frac{\partial a_{1}}{\partial b} \lambda+\frac{\partial a_{0}}{\partial b}=0 .
$$

Then, at $a_{2} a_{1}=a_{0}$ we have $\lambda= \pm i \omega_{0}$, which by (2.3) gives rise to

$$
\left.\frac{\partial(\operatorname{Re} \lambda)}{\partial b}\right|_{a_{2} a_{1}=a_{0}}=-\frac{k_{0}}{2\left(a_{2}^{2}+a_{1}\right)(c+m)} \neq 0 .
$$

Notice that $a_{2}$ and $a_{1}$ from the expression of $k_{0}$ are expressed at $b=b_{0}$. Thus, a Hopf bifurcation occurs at $B$ and $C$, which is non-degenerate if $l_{1}(0) \neq 0$, respectively, degenerate if $l_{1}(0)=0$. An expression of $l_{1}(0)$ is complex. It can be obtained easier in particular cases of the parameters.

Remark 2.5. Since the first coordinate of $C\left(-\sqrt{\alpha}, \frac{a c+1}{c+m}, \sqrt{\alpha} \frac{1-a m}{c+m}\right)$ is negative, the behavior of the model around $C$ does not present economic relevance.

Remark 2.6. The existence of Hopf bifurcation at $a_{2} a_{1}=a_{0}$ enriches the economic interpretation of the model due to stable periodic orbits which are born by the bifurcation.

2.4. Economic interpretation around $B$. Three main situations should be considered here based on the corresponding type of $B$.

1) $B$ is an attractor, that is, $a_{2}>0$ and $a_{2} a_{1}>a_{0}$. We impose also $1-a m<0$ in order to have $B$ with positive coordinates. In this case the values of the real interest rates, investments and the inflation rate are different from zero and converge to a point that has the characteristic of stability, the equilibrium $B$. This is a stable node or focus (attractor). From an economic point of view, this is a situation of interest and the tendency to reach such a steady state should be encouraged [2].

A numerical example with $B$ attractor can be obtained for $a=2.26, c=1 / 2$ and $m=1 / 2$. $B$ is well-defined in this case with positive coordinates if $0<b<0.469$. A Hopf bifurcation occurs at $b \simeq 0.23$ when $B$ changes its stability and type: it is a saddle-focus on $0<b<0.23$, respectively, an attractor on $0.23<b<0.469$. For example, at $b=0.4$, the eigenvalues of $B(0.38,2.13,0.05)$ are $-0.11 \pm 0.59 i$ and -0.80 , thus, $\operatorname{Re}\left(\lambda_{i}\right)<0$ for all $i=1,2,3$. In Figure 5 we see a bunch of orbits starting close to $B$ and $C$ and spiraling towards the points.

Remark 2.7. Since the coordinates of $B$ are all strictly positive and each of them can be chosen far from 0 , the economic relevance of the model around an attractor $B$ is more significant than around an attractor $A$, since an orbit $(x(t), y(t), z(t))$ staying around $B$ will not meet $x(t) \leq 0$, while an orbit oscillating around $A$ may enter such a zone.

2) $B$ is a saddle with $l_{1}(0)<0$. Then, $\operatorname{dim}\left(W^{s}\right) \geq 1$ and $\operatorname{dim}\left(W^{u}\right) \geq 1$. If an orbit starts with an initial value $u_{0} \in W^{s}$, there is a tendency of economic stabilization since $(x(t), y(t), z(t))$ converges to $B$ for $t$ increasing, but if it starts at $u_{0} \in W^{u}$, the economy will depart from the steady state $B$, thus $x(t)$ may become larger and larger. However, since the model has a Hopf bifurcation at $B$ and $l_{1}(0)<0$, then similar to $A$, a stable 

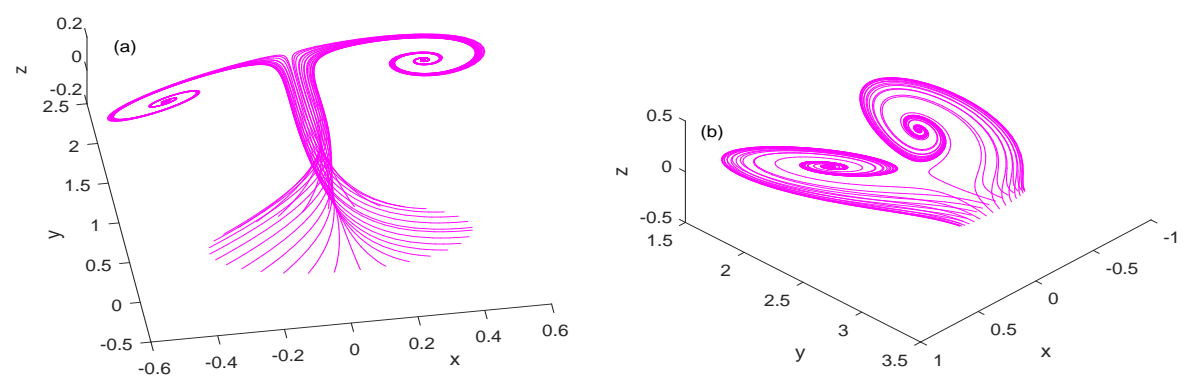

FIGURE 5. $B$ and $C$ are attractors of type focus for $a=2.26, b=0.4, c=0.5$ and $m=0.5$. The pictures show more orbits spiraling to $B$ and $C$.

periodic orbit $\Gamma_{2}$ (limit cycle) is born around $B$ when the bifurcation parameter $b$ crosses the value $b_{0}$ and $\left|b-b_{0}\right|$ is sufficiently small. All orbits starting in a small neighborhood of $\Gamma_{2}$ (including $W^{u}$ ) will tend to $\Gamma_{2}$ for $t$ increasing.

In Figure 6 we present numerically such a stable periodic orbit formed around the unstable equilibrium $B$. We consider $a=2.26, b=0.1, c=0.5$ and $m=0.5$ which lead to $B(0.88,2.13,0.11)$ with the eigenvalues $0.07 \pm 1.3 i$ and -0.88 . Thus, $\operatorname{dim}\left(W^{s}\right)=1$ and $\operatorname{dim}\left(W^{u}\right)=2$. Figure 7 (a) shows the time series of the periodic orbit $\Gamma_{2}$ depicted in Figure 6 , from which we see that the periodic orbit enters the zone with negative values for $x(t)$ and $z(t)$.
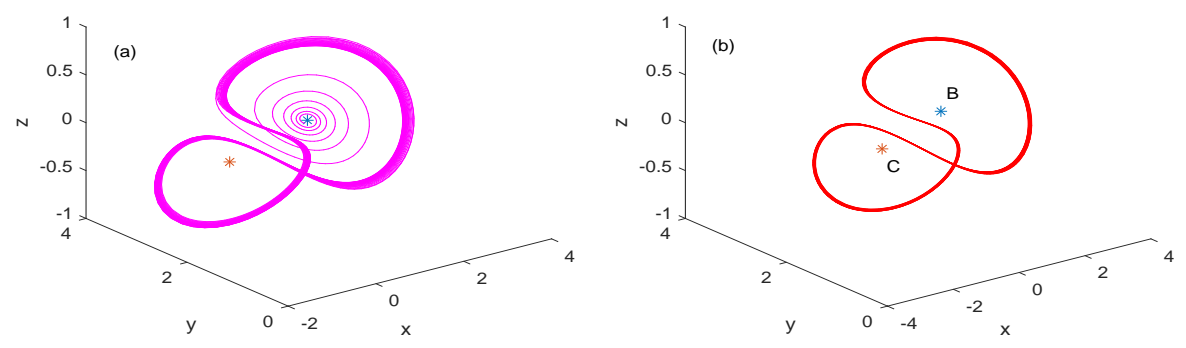

FIGURE 6. The figures are obtained for $a=2.26, b=0.1, c=0.5$ and $m=0.5$. (a) An orbit starting close to $B$ at $(0.8,2.1,0.1)$ approaches the periodic orbit $\Gamma_{2}$. (b) Approximation of a stable periodic orbit $\Gamma_{2}$ surrounding the unstable (saddle-focus) $B$. The orbit was obtained with the starting point $(2.1,2.15,0.67)$.

3) $B$ is a saddle with $l_{1}(0)>0$. Then no stable periodic arises around $B$ by the Hopf bifurcation and an orbit starting at a point $u_{0} \notin W^{s}$ will depart from the steady state $B$. From an economic point of view, this steady state is not advisable since no predictability can be achieved. For example, an increasing interest rate $x(t)$ beyond $\sqrt{\alpha}$ will decrease aggregate demand, this phenomenon being a tightening of monetary policy [9]. At the same time, a decreasing interest rate $x(t)$ beyond $\sqrt{\alpha}$ will generate an increase in aggregate demand, which may lead to a relaxation of monetary policy [12].

Remark 2.8. We notice that the system (1.1) proposed in this work has two equilibrium points with economic relevance, that is, $A$ and $B$, while the initial system studied in [10], which corresponds to $m=0$ in (1.1), has only $A$ as steady state. On the other hand, as it can be seen from the subsections 2.3 and 2.4, the new point $B$ enriches the economic relevance of the system (1.1) in this new framework with $m>0$. 

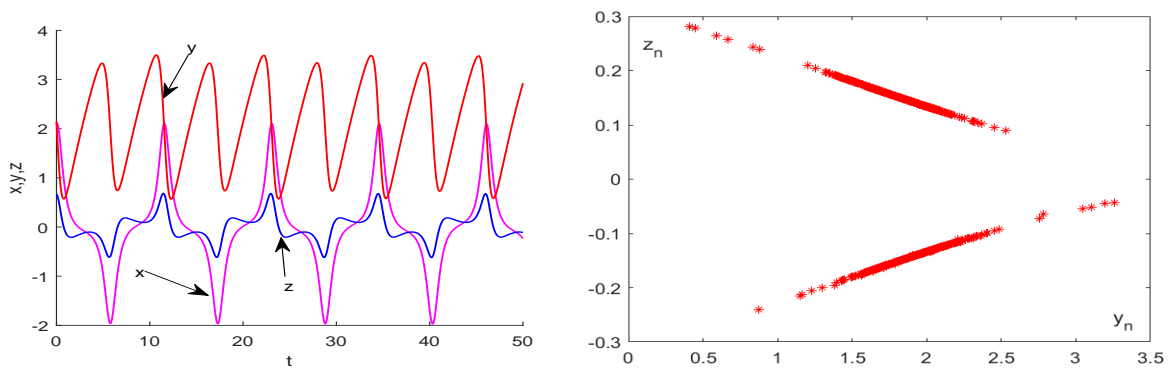

FIGURE 7. (a) The time series of the periodic orbit $\Gamma_{2}$ starting at $(2.1,2.15,0.67)$ and parametric values $a=2.26, b=0.1, c=0.5, m=0.5$. (left); (b) The values $\left(y_{n}, z_{n}\right)$ where orbits starting nearby the periodic orbit $\Gamma_{2}$ intersect the section $x=0$ (right).
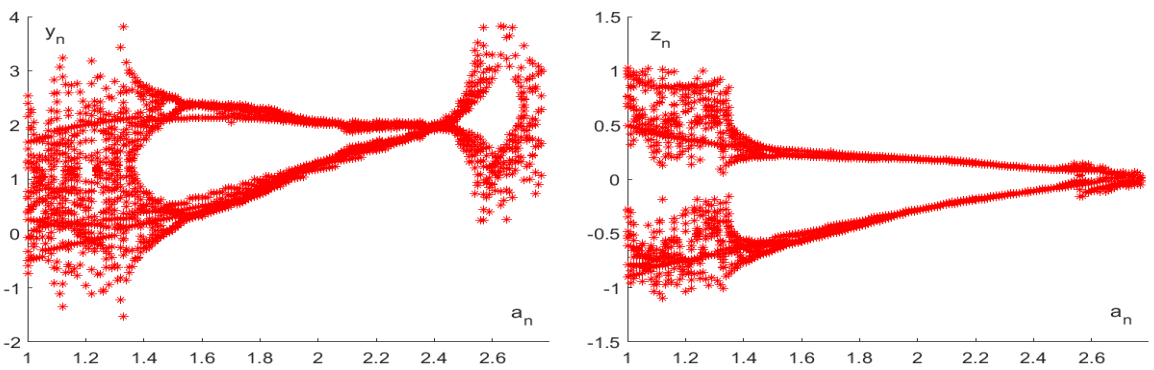

FIGURE 8. The discrete values $y_{n}$, respectively, $z_{n}$ on the Poincare section $x=0$ of the orbit starting at $(2.1,2.15,0.67)$ when $a$ ranges from 1 to 3 with a step of 0.01 . The other parametric values are fixed at $b=0.1, c=0.5, m=0.5$.

2.5. Discrete dynamics. Insights on the local behavior of the model around a periodic orbit can be obtained by using discrete dynamics, more exactly, by studying the recurrent flow's intersection with a surface (Poincare section) transversal to the flow, that is, defining a Poincaré map. As cross-section we take the surface $S=\left\{(x, y, z) \in \mathbb{R}^{3}, x=0\right\}$. Since $\left.\frac{d x}{d t}\right|_{x=0}=z$, all orbits $\gamma(t)=(x(t), y(t), z(t))$ with $z(t) \neq 0$ at $x(t)=0$, cross transversally the section $S$. In Figure 7 (b) we determine the points $\left(y_{n}, z_{n}\right)$, where more orbits starting nearby the periodic orbit $\Gamma_{2}$ at points of the form $\gamma_{0}(s)=\left(2.1-s, 2.15-\frac{s}{2}, 0.67-\frac{s}{4}\right)$, with $s$ ranging from 0 to 2 in step of 0.1 , intersect the cross-section $S$. While $y_{n}>0$, we notice that the flow intersects $S$ at positive and negative values of $z_{n}$. The code in Matlab (version R2020b) we used to obtain Figure 7 (b) is below (left).

In Figure 8, we determine the values $y_{n}$ and $z_{n}$ where an orbit starting at $\gamma_{0}(0)$ intersects $S$, when $a$ ranges from 1 to 3 in step of 0.01 , that is, $a=a_{n}=1+(n-1) \cdot 10^{-2}, n \geq 1$, while the other parameters are fixed at $b=0.1, c=0.5$ and $m=0.5$. In Figure 9 we find the same values $y_{n}$ and $z_{n}$, but when $b$ varies. The code in Matlab when $a$ varies, Figure 8 , is presented below (right). For Figure 9 the code is similar. 


\begin{tabular}{|c|c|}
\hline $\begin{array}{l}\text { clear; } a=2.26 ; b=0.1 ; c=0.5 ; m=0.5 \\
\text { for } s=0: 0.1: 2 \\
y 0=[2.1-s, 2.15-s / 2,0.67-s / 4] \\
{[t, y]=\text { ode } 45\left(@ \text { system, }\left[0: 0.1: 10^{3}\right], y 0\right)} \\
n=\text { size }(t)-1 ; \\
\text { for } i=1: n(1) \\
\text { if }(y(i, 1) * y(i+1,1)<=0) \\
\text { plot }\left(y(i, 2), y(i, 3),{ }^{\prime} r *^{\prime}\right) ; \text { hold on; } \\
\text { end } \\
\text { end } \\
\text { end } \\
\text { \%define the function 'system'; } \\
\text { function } d y=\text { system }(t, y) \\
a=2.26 ; b=0.1 ; c=0.5 ; m=0.5 ; \\
d y=z \operatorname{eros}(3,1) ; \\
d y(1)=y(3)-a * y(1)+y(1) * y(2) \\
d y(2)=1-b * y(2)-y(1) * y(1) ; \\
d y(3)=-y(1)-c * y(3)+m * y(1) * y(2) ; \\
\text { end }\end{array}$ & $\begin{array}{l}\text { clear; } b=0.1 ; c=0.5 ; m=0.5 ; \\
\text { for } a=1: 0.01: 3 \\
y 0=[2.1,2.15,0.67] \\
{[t, y]=\text { ode } 45(@(t, y) \operatorname{system}(t, y, a)} \\
[0: 0.1: 100], y 0) ; \\
n=\operatorname{size}(t)-1 ; \\
\text { for } i=1: n(1) \\
\text { if }(y(i, 1) * y(i+1,1)<=0) \\
\text { plot }\left(a, y(i, 2),{ }^{\prime} r *^{\prime}\right) ; \text { hold on; } \\
\text { end } \\
\text { end } \\
\text { end } \\
\% \text { define a function 'system' on } a ; \\
\text { function } d y d t=s y s t e m(t, y, a) \\
b=0.1 ; c=0.5 ; m=0.5 ; \\
d y d t=z e r o s(3,1) ; \\
d y d t(1)=y(3)-a * y(1)+y(1) * y(2) ; \\
d y d t(2)=1-b * y(2)-y(1) * y(1) ; \\
d y d t(3)=-y(1)-c * y(3)+m * y(1) * y(2) ; \\
\text { end }\end{array}$ \\
\hline
\end{tabular}

The Matlab codes for obtaining the Figures 7 (b), 8, 9 .
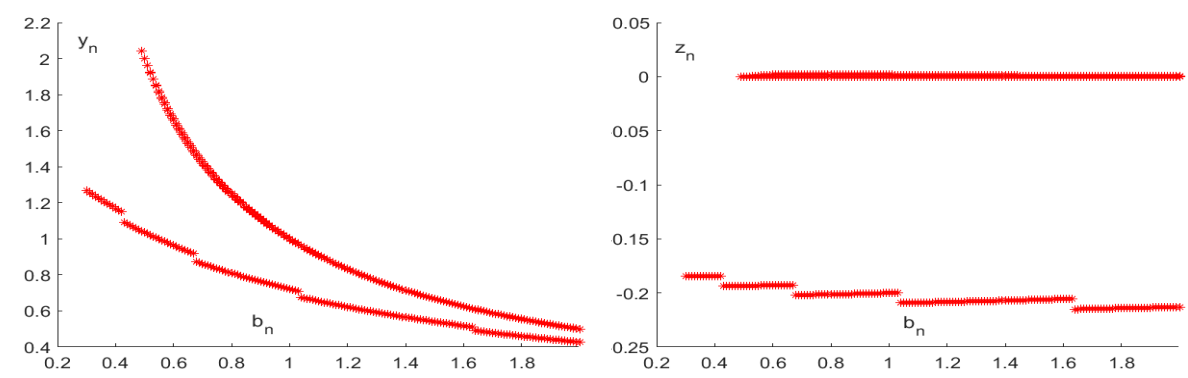

FIGURE 9. The discrete values $y_{n}$, respectively, $z_{n}$ on the Poincaré section $x=0$ of the orbit starting at $(2.1,2.15,0.67)$ when $b$ ranges from 0.3 to 2 with a step of 0.01 . The other parametric values are fixed at $a=2.26, c=0.5, m=0.5$.

\section{CONCLUSiOnS}

Often, complex economic systems seem too chaotic to be able to recognize in them a certain typology. However, the use of differential equations for studying dynamic economic systems allows the identification of useful elements for economic decision-makers [5], [13]. The representation of dynamic economic systems in the form of graphs may indicate that there is a certain state in which the system is trying to reach. The objective characteristics of the economic-social environment, correlated with the expectations of the economic agents, can provide important and relevant signals on the evolution of economic systems. Therefore, the economic decision-makers should take into account the evolution of the dynamic systems so as to favor the creation of stable equilibrium situations, as well as to avoid the emergence of unstable regions. 
Acknowledgments. This research was supported by Horizon2020-2017-RISE-777911 project.

\title{
REFERENCES
}

[1] Akram, T. The Japanese economy: stagnation, recovery, and challenges. Journal of Economic Issues 53 (2019), no. 2, 403-410.

[2] Angeletos, G. M.; Calvet, L. E. Idiosyncratic production risk, growth and the business cycle. J. Monet. Econ. 53 (2006), no. 6, 1095-1115.

[3] Bernheim, B. D.; Thomadsen, R. Memory and anticipation. Economic Journal 115 (2005), no. 503, $271-304$.

[4] Chen, L. H. A model for ex ante real interest rates. Applied Economics Letters 8 (2001), no. 11, 713- 718.

[5] Du, J.; Huang, T.; Sheng, Z. Analysis of decision-making in economic chaos control. Nonlinear Anal. Real World Appl. 10 (2009), no. 4, 2493-2501.

[6] Eggertsson, G. B.; Singh, S. R. Log-linear approximation versus an exact solution at the ZLB in the new keynesian model. J. Econ. Dyn. Control 105 (2019), 21-43.

[7] Favara, G.; Song, Z. House price dynamics with dispersed information. J. Econ. Theory 149 (2014) $350-382$.

[8] Hasui, K.; Nakazono, Y.; Teranishi, Y. Role of expectations in a liquidity trap. J. Jpn. Int. Econ. 52 (2019), 201-215.

[9] Hommes, C.; Lustenhouwer, J. Managing unanchored, heterogeneous expectations and liquidity traps. J. Econ. Dyn. Control 101 (2019), 1-16.

[10] Ma, J. H.; Chen, Y. S. Study for the bifurcation topological structure and the global complicated character of a kind of nonlinear finance system. Applied Mathematics and Mechanics-English edition 22 (2001), no. 11, 1240-1251.

[11] Nakata, T.; Schmidt, S. Conservatism and liquidity traps. J. Monet. Econ. 104 (2019), 37-47.

[12] Tsuruga, T.; Wake, S. Money-financed fiscal stimulus: The effects of implementation lag. J. Econ. Dyn. Control 104 (2019), 132-151.

[13] Wu, W.; Chen, Z.; Ip, W. H. Complex nonlinear dynamics and controlling chaos in a Cournot duopoly economic model. Nonlinear Anal. Real World Appl. 11 (2010), no. 5, 4363-4377.

\author{
DEPARTMENT OF MATHEMATICS \\ POLITEHNICA UNIVERSITY OF TIMIŞOARA \\ PTA Victoriei, No. 2, 300006 TimişOARA, ROMANiA \\ Email address: gheorghe. moza@upt.ro \\ Email address: loredana. ciurdariu@upt.ro \\ DEPARTMENT OF MANAGEMENT \\ POLITEHNICA UNIVERSITY OF TIMISSOARA \\ PtA Victoriei, No. 2, 300006 TimişOARA, RomAniA \\ Email address: eugenia.grecu@upt.ro
}

